Ann. Zootech., I978, 27 (I), 6I-74.

\title{
Utilisation du Monensin dans la ration des ruminants. \\ II. - Effets sur les fermentations et la population microbienne du rumen
}

\author{
J. P. JOLANY et J. SENAUD
}

avec la collaboration technique de M. P. GIRARD, A. VIzET et J. IEririri:

\author{
Station de Recherche's sur l'Élevage des Ruminants, \\ Centre de Recherches de Clermont-Ierrand, I.N.R.A., \\ Theix, 631 Bo Beamont, France \\ Laboratoire associé au C.N.R.S. no 138 , \\ (Biologie Comparée des Protistes), \\ Université Clermont II, Les Cézeaux \\ B.P. $+5,63170$ Aubière (France).
}

\section{Résumé}

Nous avons étudié la digestion dans le rumen de moutons, d'une ration constituée en proportion constante $(60: 40)$ de foin de prairie naturelle distribuée sous forme hacliée et d'aliment condensé contenant o ou 20 ou 40 p.p.m. de Monensin (antibiotique issu de: "Streptomyces cinnamonensis "). L'aliment aggloméré comportait des pulpes de betteraves déshydratées (50 p. cent), de la luzerne déshydratée (25 p. cent) et une part réduite de céréales ( 13,5 p. cent) associée à du tourteau de soja (Io p. cent).

La présence de Monensin dans la ration provoque une augmentation (environ 30 p. cent) de la proportion molaire d'acide propionique, alors que la somme des acides gras volatils a significativement diminué. Parallèlement, nous avons montré un accroissement de la concentration du jus de rumen en acide lactique. Enfin, la composition des gaz du rumen indique que la réduction de la méthanogénèse est importante lorsque les animaux reçoivent du Monensin.

I'ensemble de ces résultats peut s'expliquer par une modification importante de l'écosystème que constitue le rumen, à la suite de l'ingestion de Monensin par les animaux. Nous avons en effet observé une diminution significative de la biomasse de protozoaires ciliés du rumen ainsi qu'une augmentation du nombre des bactéries dont la taille est supérieure à I us qui ne représentent, cependant, qu'une partie de l'ensemble des bactéries du rumen.

\section{Introduction}

De nombreux travaux ont montré que l'incorporation d'antibiotiques dans. les aliments se traduisait par une augmentation des performances zootechniques. des ruminants (BEEson et al., I957; DYer, Exsminger et BLUE, I957; Kor ARI et 
al., Ig60; KLOPFENSTEIN, PURSER et TYzNiK, Ig64) sans provoquer de troubles digestifs (NEUMAN, SNAPP et GaLL, I95I). La présence d'antibiotiques dans la ration modifie la composition de la population microbienne du rumen (HUNGATE, FLETCHER et DYER, I955) et par conséquent, la nature et la quantité des produits terminaux de la digestion. Ainsi, la tylosine et l'auréomycine semblent favoriser le développement des protozoaires dans le rumen et orienter les fermentations vers une production accrue d'acide propionique, tandis que l'acidité totale du milieu diminue (Purser, Kl,opfenstein et Ci,INe, i965).

Ies études récentes (RICHARDSON et al., I974) ont montré qu'un antibiotique (Monensin), issu d'une souche de Streptomyces cinnamonensis (HANEY et HoEHN, I 967), connu pour ses propriétés anticoccidiennes et son action antibiotique sur les bactéries Gram - , modifie d'une manière importante les fermentations dans le rumen; la production d'acide propionique est accrue de $52 \mathrm{p}$. cent lorsque les bovins en reçoivent $200 \mathrm{mg} / \mathrm{j}$. A partir de cette observation, l'incorporation de Monensin dans l'alimentation du Ruminant a été testée aux U.S.A. sur un nombre important de bovins à l'engrais. L'ensemble des résultats indique que pour une teneur de 22 à 44 p.p.m. dans des rations généralement riches en céréales ou en fourrages verts, le Monensin réduit la quantité ingérée d'environ I5 p. cent sans modifier la croissance des animaux (RAUN et al., I974). La composition des carcasses n'est pas modifiée (POTTER et al., I 974a), que ce soit pour des régimes riches en énergie (BRowx et al., I974) ou lorsque les animaux sont au pâturage(PotTER et al., I974b).

Cependant, nous connaissons peu 1'action du Monensin lorsqu'il est incorporé à des rations plus riches en fourrages secs, du type de celles généralement utilisées en France. C'est pourquoi des essais ont été entrepris à 1'I.N.R.A. pour étudier l'action du Monensin sur la vitesse de croissance, l'efficacité alimentaire et la qualité des carcasses de taurillons. GEAY et BERANGER (I977) ont montré que la présence de Monensin dans la ration des taurillons augmente l'efficacité alimentaire sans modifier la croissance et la composition des carcasses. Nous avons réalisé une étude complémentaire sur des moutons recevant des régimes voisins, afin de déterminer les modifications éventuelles de la population microbienne dominante dans le rumen ainsi que des produits terminaux de son métabolisme.

\section{Matériel et méthodes}

\section{Régimes et animaux}

Les animaux ayant refusé d'ingérer l'aliment condensé seul, utilisé par GEAY et BÉRANGER, nous avons dû utiliser trois régimes constitués en proportion constante $(60: 40)$ de foin de prairie naturelle et d'aliment concentré qui contenait o- 20 et 40 p.p.m. de Monensin (tabl. I). Chacun des régimes a été distribué, selon un dispositif en carré latin, à 3 moutons adultes de race Texel pesant environ $40 \mathrm{~kg}$, munis de fistule permanente du rumen. Chaque période a duré, au total, environ I mois; 3 semaines ont été consacrées à l'adaptation des animaux à chaque régime tandis que les mesures ont été effectuées au cours de la $4^{\mathrm{e}}$ semaine. 


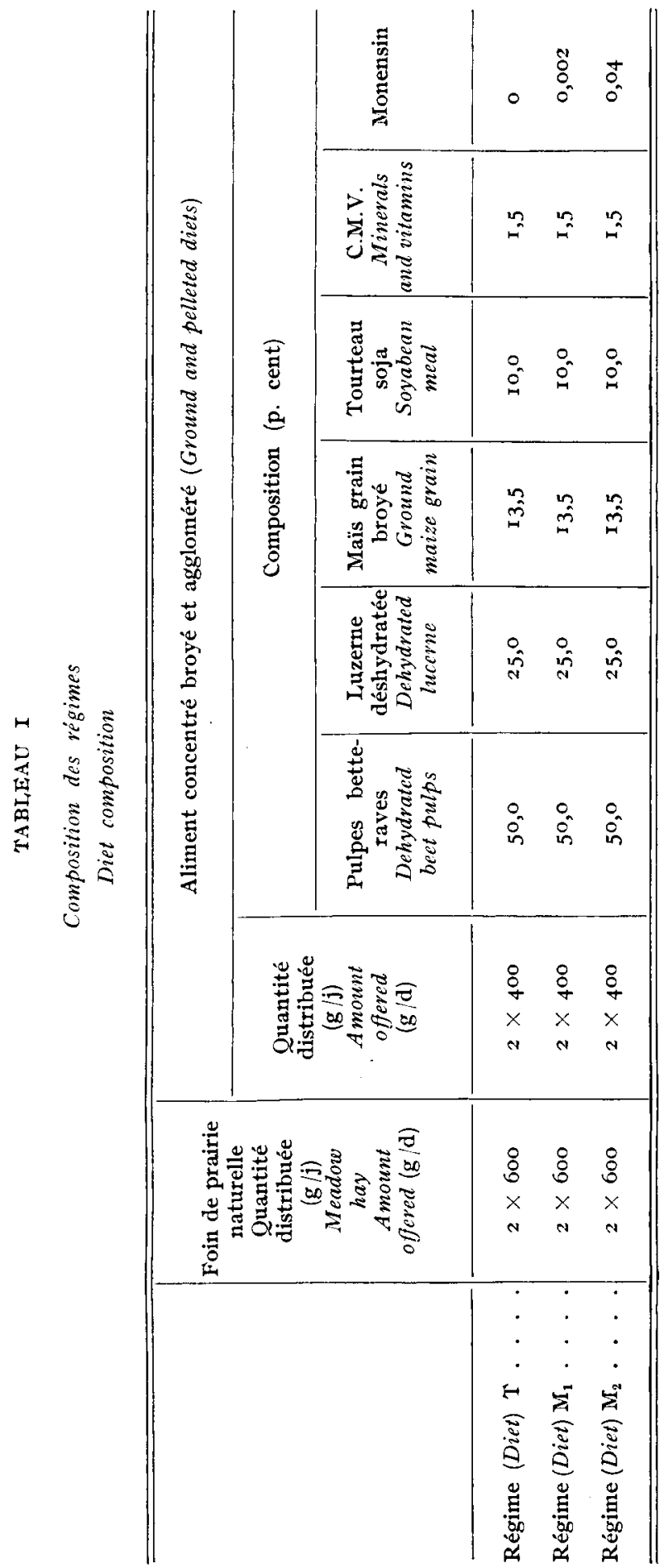




\section{Prélèvements et mesures}

Les quantités d'aliments ingérées par les animaux et les refus ont été mesurés tous les jours pendant la durée de l'expérience. Pendant deux jours consécutifs, nous avons prélevé environ roo $\mathrm{m} 1$ de contenu de rumen au fond $\mathrm{du}$ sac ventral à différents temps après le début $d u$ repas. I,e contenu a été ensuite filtré sur deux couches de gaze pour effectuer les mesures suivantes:

- Sur le filtrat prélevé juste avant le repas, une demi-heure puis toutes les heures jusqu'à $8 \mathrm{~h}$ après le début du repas, nous avons déterminé le pH puis dosé la concentration totale et la proportion des différents acides gras volatils par chromatographie en phase gazeuse (RIGAUd et JouRNE'T, I970).

- L'acide lactique a été dosé sur le filtrat prélevé juste avant le repas, une demi-heure, une heure et deux heures après le début du repas (BARkER et SumMERSON, I94I).

- La concentration des principales espèces de protozoaires et de bactéries a été déterminée dans le filtrat prélevé une heure après le début du repas, tous les jours, pendant au moins une semaine. Les protozoaires du rumen ont été identifiés et comptés sous une loupe binoculaire dans $0,05 \mathrm{ml}$ de jus filtré grossièrement sur une grille métallique dont les mailles mesurent environ $2 \mathrm{~mm}$. Le liquide est déposé dans une cuve de Dolıfouss à l'aide d'une pipette dont l'extrémité a été sciée selon les recommandations de SAUvanT et Gove'T (I970). On ajoute un volume suffisant de liquide de Hungate à $39^{\circ} \mathrm{C}\left(\mathrm{NaCl} 6 \mathrm{~g}-\mathrm{Na} \mathrm{H} \mathrm{CO}_{3}\right.$ I g $-\mathrm{H}_{2} \mathrm{KPO}_{4}$ I g $\mathrm{Mg} \mathrm{SO} \mathrm{S}_{4}$, I $\left.\mathrm{g}-\mathrm{Ca} \mathrm{Cl}_{2} \mathrm{O}, \mathrm{I} \mathrm{g} / 1\right)$ pour couvrir l'ensemble des unités de comptages de la cuve dont une sur deux sera examinée. Les bactéries ont été comptées en cuve tubulaire à plancton WIr. de Io $\mathrm{ml}$ à l'aide du microscope biologique inversé WIL,D M 40 .

Nous avons également déterminé la composition des gaz du rumen prélevés pendant 2 jours consécutifs juste avant le repas, une heure et deux heures après, par ponction dans le sac dorsal à l'aide d'une seringue munie d'un robinet, après s'être assuré que la canule n'avait pas été ouverte depuis au moins 24 heures. L'analyse de l'échantillon gazeux a été effectuée par chromatographie gaz-solide à l'aide de deux colonnes disposées en série, séparées par une troisième colonne vide, montées sur un chromatographe en phase gazeuse muni d'un détecteur à conductibilité thermique ('TACU, données non publiées). La première colonne est remplie de gel de silice (60-80 mesh) de façon à séparer les pics d'azote et d'oxygène et à obtenir en fin d'analyse le pic correspondant au gaz carbonique.

\section{Résultats}

Les animaux ont bien ingéré l'aliment concentré lorsque celui ci était accompagné de foin, mais l'un d'eux a refusé systématiquement d'ingérer la totalité de la ration qui lui était distribuée et pour cette raison, nous avons dû l'éliminer au cours d'une période expérimentale (régime $\mathrm{M}_{2}$ ) afin que les résultats soient comparables entre animaux. 


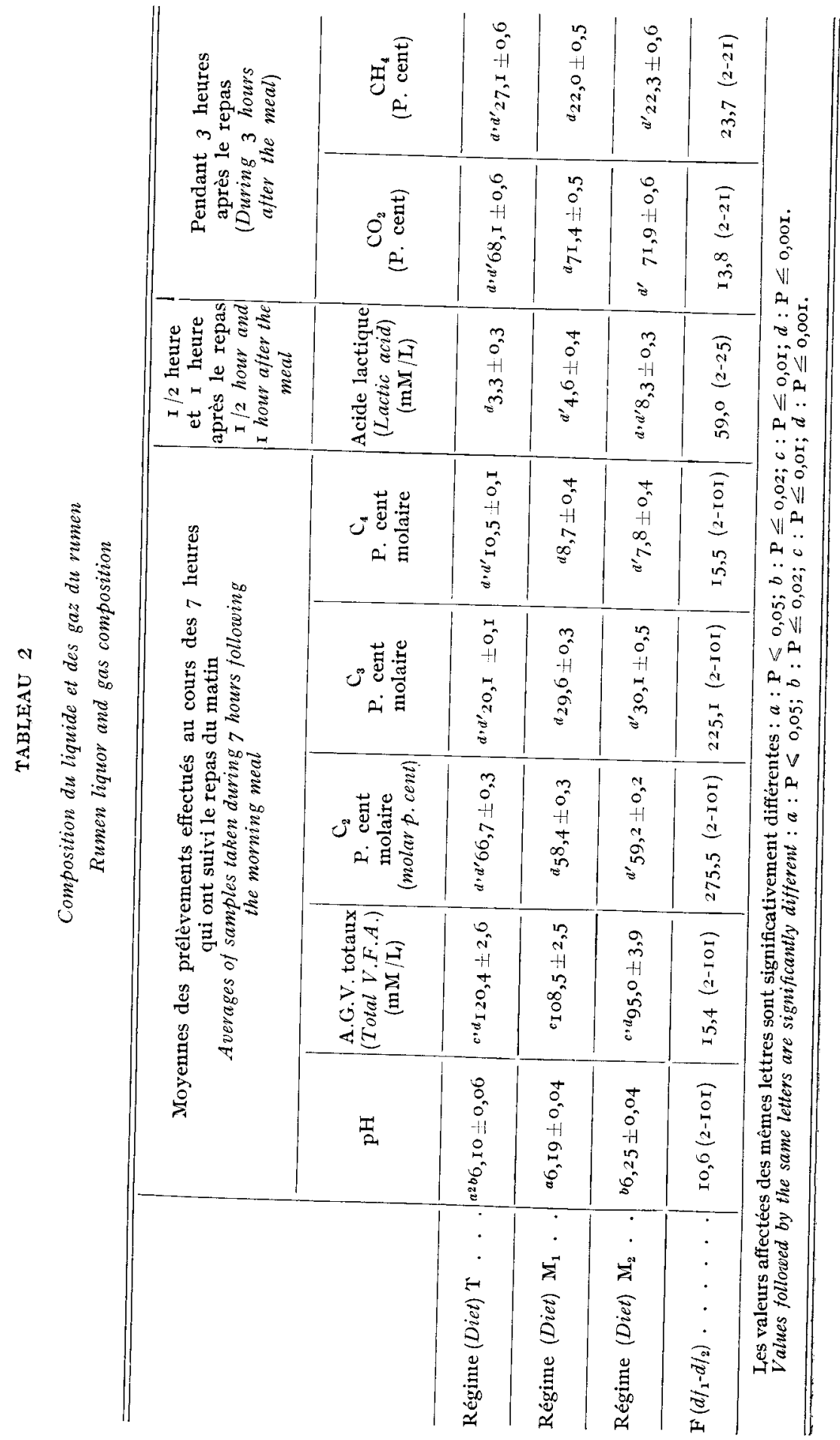


Fermentations dans le rumen

$p H$ (fig. I).

Avec les rations contenant du Monensin, le pH du jus de rumen a toujours été significativement plus élevé (tabl. 2). Les différences observées entre les deux niveaux, pendant les trois premières heures qui ont suivi le repas, n'ont pas été significatives. C'est 7 heures après le début du repas que le seuil de signification $(\mathbf{P} \leqslant \mathrm{O}, \mathrm{OI})$ est devenu le plus important.

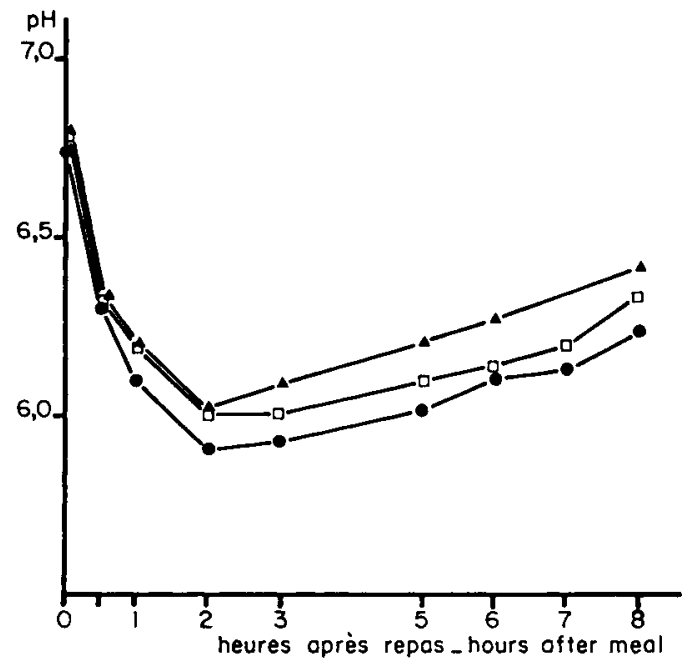

FIG. I. - $p H$ du jus de rumen.

$p H$ of the rumen fluid.

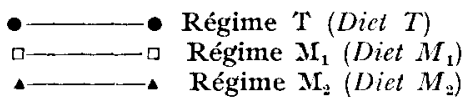

Acides gras volatils

La concentration du jus de rumen en acides gras volatils totaux a été réduite lorsque les animaux ont ingéré du Monensin avec leur ration. Cette diminution a été d'autant plus importante que la teneur en Monensin de l'aliment était plus élevée (fig. 2, tabl. 2).

La présence du Monensin a diminué la proportion molaire des acides acétique et butyrique; corrélativement, la proportion d'acide propionique a été fortement accrue (tabl. 2). On observe aucune différence dans la teneur en acide propionique du jus de rumen pour les doses de Monensin étudiées. Il semble donc que l'effet maximum soit déjà atteint, au moins en ce qui concerne la formation d'acide propionique, lorsque les animaux ingèrent 20 p.p.m. de Monensin, soit $8 \mathrm{mg}$ par sepas pour des moutons pesant environ $40 \mathrm{~kg}$ (fig. 2). 

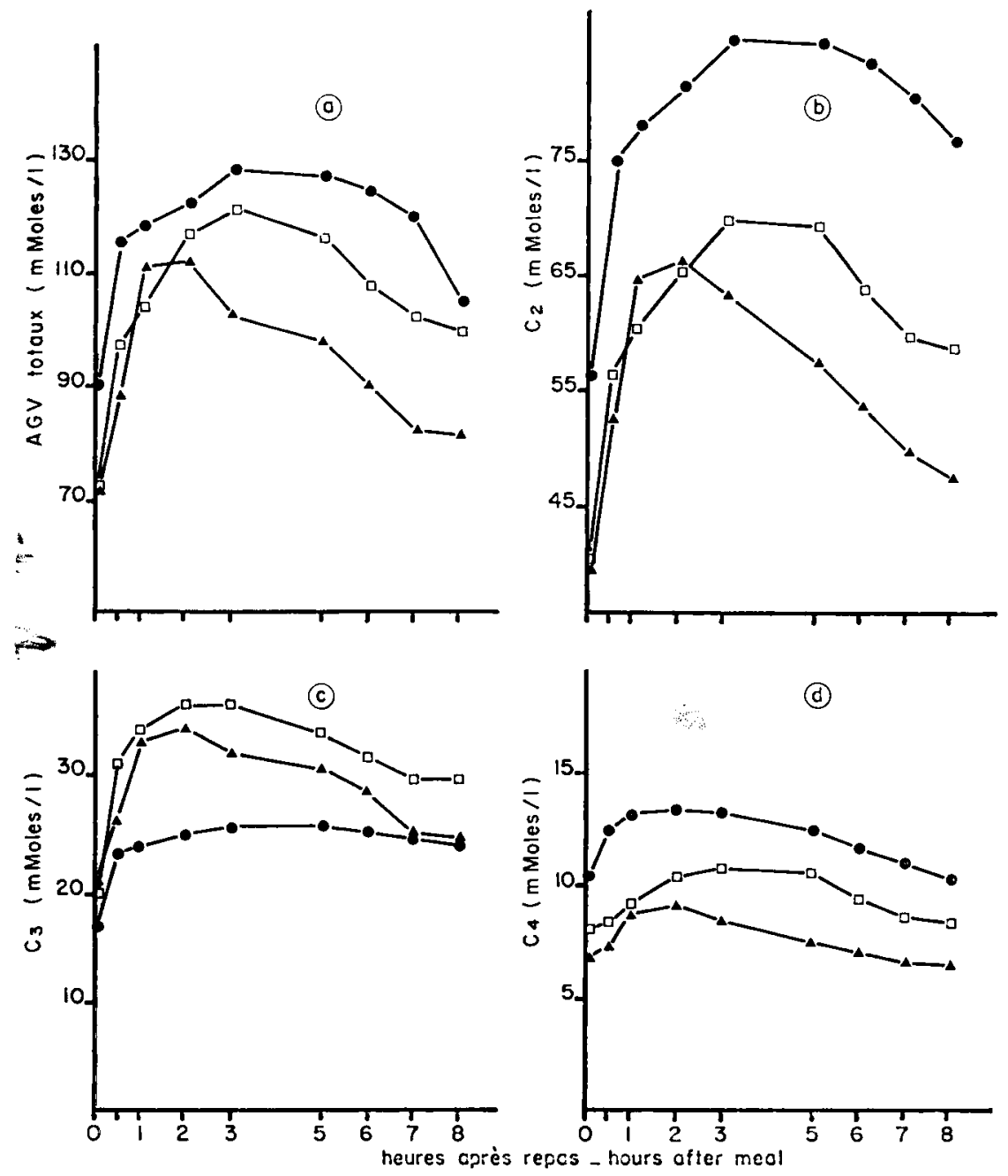

FIG. 2. - Concentration en acides gras volatils du jus de rumen

Volatile fatty acids in rumen fuid

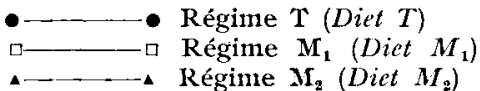

\section{Acide lactique}

La concentration en acide lactique du jus de rumen dans l'heure qui suit le début du repas a augmenté avec la teneur en Monensin de la ration (tabl. 2). Les différences sont hautement significatives entre les trois régimes étudiés. I.'acide lactique a été ensuite rapidement métabolisé, puisque deux heures après le début du repas, sa concentration dans le jus de rumen est négligeable. 


\section{Gaz du rumen}

La présence de Monensin a provoqué une forte diminution de la proportion de méthane dans le mélange des gaz du rumen, et corrélativement, une augmentation de la teneur en gaz carbonique. Cette différence, très nette lorsque les animaux sont à jeun, se maintient après le début du repas (fig. 3).

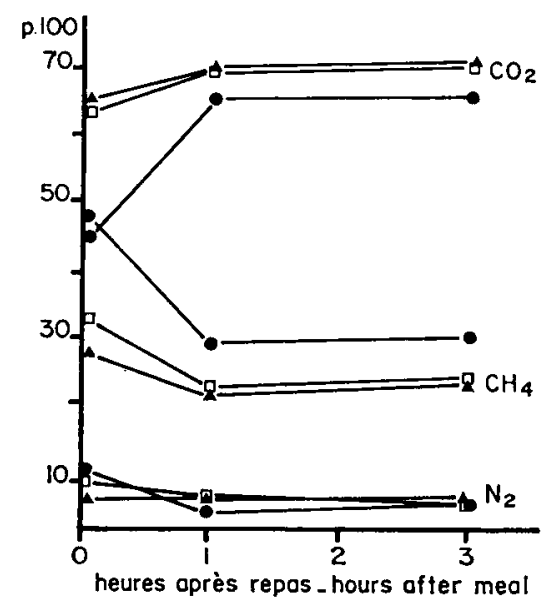

FIG. 3. - Composition des gaz du rumen Rumen gas composition

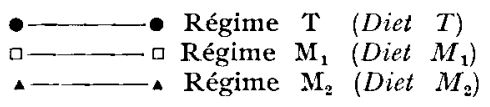

\section{Population microbienne du rumen}

\section{Les protozoaires}

La concentration totale des protozoaires ciliés dans le liquide du rumen a diminué d'une manière non significative lorsque les animaux ont reçu les régimes contenant du Monensin (fig. 4a). La population d'Entodinium sp. qui est largement dominante dans le rumen de ces moutons a évolué de la même façon (tabl. 3). La concentration des espèces à grande taille telles que Polyplastron multivesi culatum ou Isotricha sp. a été significativement réduite en présence de Monensin. Nous avons exprimé ces résultats en unités de volume et non plus en nombre de protozoaires, sachant que Isotricha sp. et Polyplastron multivesiculatum d'une part, Dasytricha ruminantium et Entodinium sp., d'autre part, ont sensiblement la même taille, et que le volume de $P$. multivesiculatum est environ I4 fois supérieur à celui d'Entodinium caudatum (ColfmaN, I975). Nous avons supposé que la densité des ciliés est constante quelle que soit leur taille et donc que le rapport des masses est égal au rapport des volumes. Nous avons ainsi montré une diminution significative $(\mathrm{P} \leqslant \mathrm{O}, \mathrm{OI})$ de la biomasse des ciliés dans le rumen lorsque les animaux reçoivent du Monensin, qui a été d'autant plus importante que la teneur en Monensin de la ration était élevée (fig. 4 b). 


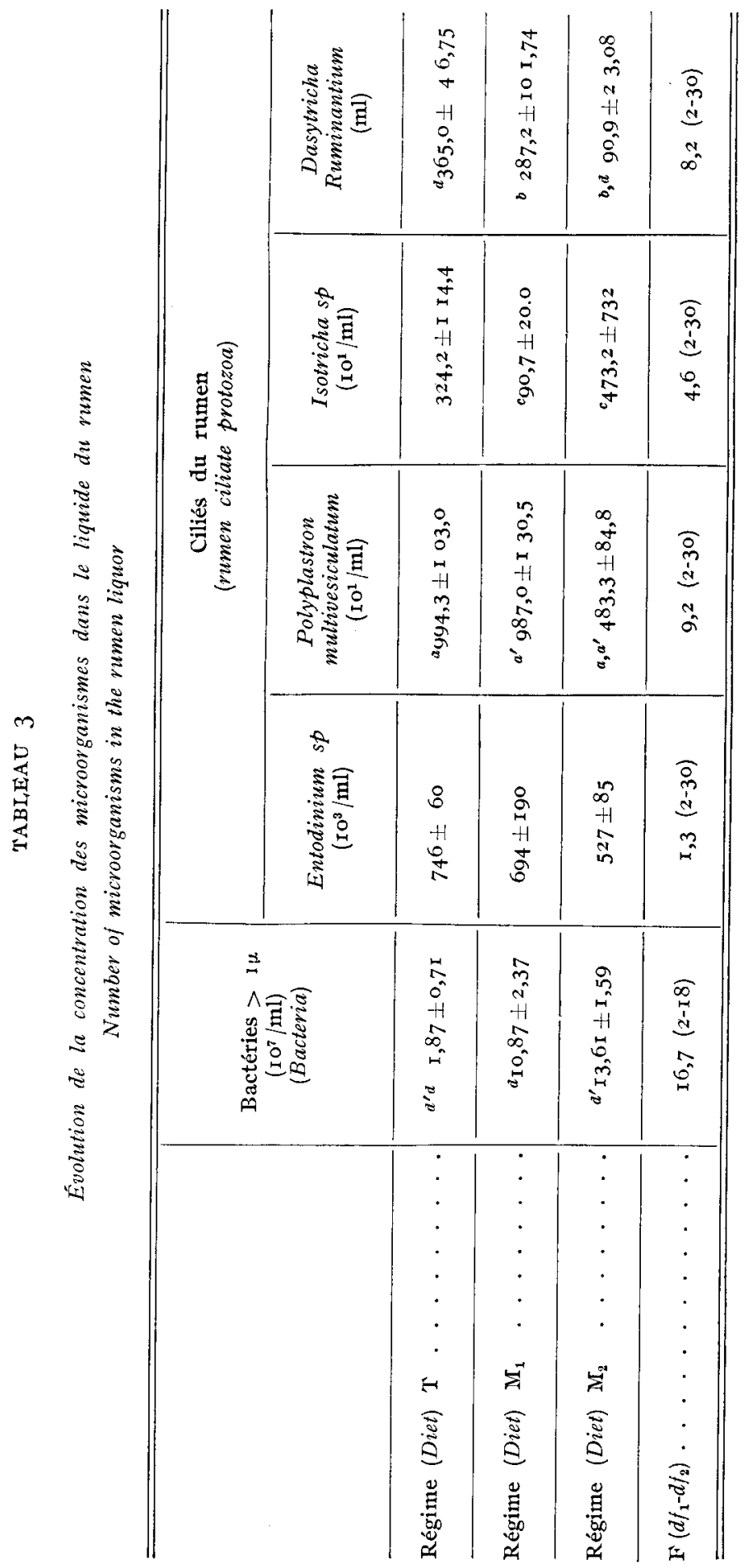


(a)

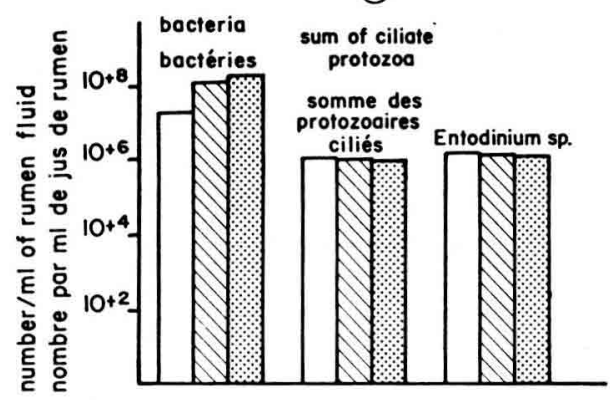

(b)

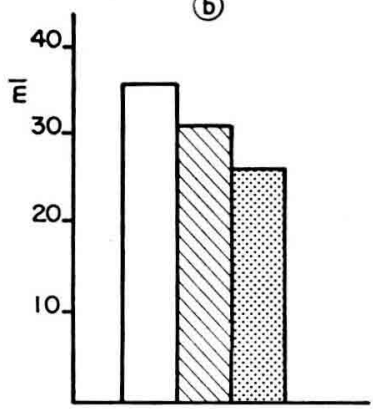

FIG. 4a. - Concentration en microorganisms du jus de vumen

Distribution of rumen microorganisms in rumen fluid

FIG. 4 b. - Volume des ciliés dans $I$ litve de jus de numen Volume occupied by ciliates in x litre of numen fluid

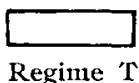

Regine ' $T$ (Diet $T$ )

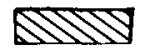

Régime $\mathrm{M}_{1}$

(Diet $M_{1}$ )

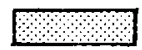

Régime $\mathrm{M}_{2}$

(Diet $M_{2}$ )

Ce résultat a été confirmé au cours d'une autre étude que nous avons effectuée sur des taurillons recevant de l'herbe fraîche (JOUANY et SENAUD, données non publiées). La population des ciliés constituée uniquement d'Entodinium $s p$. a alors brusquement diminué lorsque les animaux ont reçu du Monensin et s'est maintenue à ce niveau. Les modifications apportées par le Monensin ont été hautement significatives $(P \leqslant 0,00 r)$.

\section{Les bactéries}

La concentration dans le jus de rumen des bactéries que nous avons comptées (taille supérieure à I micron) augmente d'environ 7 fois lorsque les animaux reçoivent les rations contenant du Monensin (tabl. 3). Cependant, cette fraction de bactéries est nettement sous dominante par rapport à l'ensemble des populations bactériennes du rumen ( $10^{8}$ au lieu de $10^{9} \mathrm{à}^{10} 0^{10}$ par ml selon HUNGATE, I 966 ).

\section{Discussion}

En accord avec les résultats antérieurs (RICHARDSON et al., I974; Brown et $a l$., I974; POTTER et al., I974 $a$ et $b$; RAUn et al., I974; BEEDE et FARLIN, I975), nous avons observé que la présence de Monensin dans la ration favorisait la formation d'acide propionique dans le rumen au détriment des acides acétique et butyrique. Nous avons, de plus, montré que la concentration en acides gras volatils totaux $\mathrm{du}$ jus de rumen était réduite. Ceci doit être la conséquence d'une production plus faible plutôt que d'une augmentation de l'absorption, puisque le $\mathrm{pH} d u$ 
milieu est plus élevé lorsque les animaux reçoivent du Monensin. Nous avons également observé une réduction de la méthanogénèse et une production accrue d'acide lactique en liaison avec la présence de Monensin. L'ensemble de ces résultats traduit une modification profonde de l'écosystème que nous avons corrélativement observée contrairement aux résultats de Dinius et Simpson (I975) et de Drnius, Simpson et Marsh (1976).

La réduction de la production des acides gras volatils dans le rumen est Ja conséquence directe d'une diminution de l'activité microbienne lorsque les animaux reçoivent du Monensin. Nous avons montré que si globalement, le rombre de ciliés variait peu, leur biomasse était fortement réduite par suite de la diminution significative de la concentration des espèces à grande taille que sont $P$ oly $p$ lastron multivesiculatum et Isotricha $s p$. L'augmentation du nombre de bactéries de grande taille doit probablement traduire une évolution générale semblable de l'ensemble de la population bactérienne qui devient plus importante lorsque les protozoaires du rumen disparaissent (EADIE, I962; EAdIE et Hobson, I962; BARRINGER, 'TRENKLE et BURROUGHS, I966). Mais cette augmentation ne s'accompagne pas d'un accroissement de l'activité bactérienne lorsque la population des ciliés diminue. Kurihara et al. (I968) ont en effet montré que l'activité des bactéries est stimulée par la présence des ciliés qui les ingèrent en permanence. Il est donc vraisemblable que le métabolisme et le taux de croissance des bactéries aient été réduits, lorsqu'en présence de Monensin, la biomasse des ciliés a diminué.

De nombreuses études ont porté sur la composition du mélange des acides gras volatils en fonction de l'importance de la faune du rumen. L'évolution de l'acide propionique est variable. Selon Klopfenstein, Purser et Trznik (Ig66). Youssef et Ailien (I968), Eadie et al. (I969), Males et Purser (I970), WhiteLAW et al. (I972), une réduction de la population des ciliés ne traduit pas une augmentation de la proportion d'acide propionique. A l'opposé, les travaux de ABou Akmada et Er, Shaizy (i 964), Christiansen, Kawashima et Burroughs (i965), Kurihara et al. (I968), EAdie et GIIL (I97I), EADIE (I973) ont montré que la teneur en acide propionique diminue dans le rumen d'animaux défaunés. S'il est probable que les modifications observées au niveau de la biomasse des ciliés jouent un rôle sur la composition des acides gras volatils, l'augmentation de la production d'acide propionique observée avec les régimes contenant le Monensin est trop importante pour être expliquée par ce seul phénomène. Ellle pourrait provenir de la production accrue d'acide lactique que nous avons observée, en particulier avec le régime contenant la plus forte dose de Monensin. On sait en effet que l'acide lactique peut être métabolisé dans le rumen en acide propionique par l'intermédiaire de l'acrylate (JohNs, I95Ia; SATTER et ESDALE, I968) ou de succinate (JohNS, I95 I $b$; HoWARD, I959). L'augmentation parallèle de la concentration en acide lactique et en acide propionique du jus de rumen traduit vraisemblablement une action inhibitrice spécifique de Monensin sur certaines espèces bactériennes ou sur certaines enzymes au profit d'autres qui se développent, provoquant une réduction et une déviation des processus fermentaires dans le rumen.

Une modification du transit à la sortie du rumen pourrait également expliquer la plus grande proportion d'acide propionique dans le mélange des acides gras volatils. Selon HARRISSON et al. (1975), il existe une relation inverse entre la vitesse de renouvellement du contenu d'une part et la proportion molaire de l'acide propionique d'autre part. Or, la réduction de l'activité microbienne dans le rumen permet de penser qu'effectivement la vitesse du renouvellement du contentı à l'intérieur de cet organe a été plus faible avec les régimes contenant du Monensin. Enfin, la diminution de la production de méthane que nous avons observée lorsque 
les animaux reçoivent du Monensin est en accord avec la réduction de la formation d'acide acétique (Hungate, I966; DEMEYER et VAN NEVEL, I975).

La présence de Monensin dans le rumen, aux doses utilisées, modifie donc le profil de la population microbienne d'une façon durable puisqu'il n'y a pas eu de réduction de l'effet avec le temps. Cet anticoccidien agit par conséquent sur la nature et l'importance des produits terminaux de la digestion dans le rumen, dans un sens qui paraît favorable à une meilleure utilisation de l'énergie des aliments comme le montrent les résultats zootechniques obtenus par GEAY et BÉRANGER (I977).

Accepté pour publication en septembre 1977.

\section{Summary}

\section{Utilization of Monensin in the diets of ruminants.}

\section{II. - Effects on the fermentations and the microbial population of the rumen}

A $3 \times 3$ latin-square design involving 3 castrated sheep fitted with rumen cannulas was used. They were fed hay and pelleted diets $\left(60 ; 4^{\circ}\right)$. The ground and pelleted diet contained 50 p. cent dehydrated beet pulp, $25 \mathrm{p}$. cent dehydrated lucerne and $13.5 \mathrm{p}$. cent maize grain associated with Io p. cent soya bean meal as well as o or 20 or $40 \mathrm{ppm}$ of "Monensin $\nu$ (an antibiotic derived from Streptomyces cinnamonensis) (diets $T, \mathbf{M}_{1}$ and $\mathbf{M}_{2}$, table $\mathbf{I}$ ). Digestion in the rumen just before and after the morning meal was studied by taking samples from the ventral sac.

"Monensin" significantly increased the molar percentage of propionic acid and the $\mathrm{pH}$ in the rumen liquor (table 2 ). The total volatile fatty acid and acetic acid concentrations were lower when animals were fed "Monensin" diets (fig. $2 a, 2 b, 2 c$ ). Just after feeding we also observed an increase in the lactic acid concentration (Table 2). The rumen gas composition showed an important decrease in the proportion of $\mathrm{CH}_{4}$ and, at the same time an increase in the proportion of $\mathrm{CO}_{2}$ with diets $\mathrm{M}_{1}$ and $\mathrm{M}_{2}$ (fig. 3).

It may be concluded from these results, that "Monensin "favorably altered the rumen ecosystem as far as energy metabolism is concerned. A small, non significant decrease in the concentration of rumen ciliate protozoa (fig. $4^{a}$ ) and a significant decrease in the ciliate biomass (fig. $4 b$ ) were observed as well as a 7 fold increase in the number of large bacteria $(>\mathrm{I} \mu$ ) (table 3 ). However, no data on the number of small bacteria were available.

\section{Références bibliographiques}

A Bou AKKAUA A. R., EI, SHAZI, K., I964. Effect of absence of ciliate protozoa from the rumen on microbial activity and growth of lambs. Appl. Microbiol., 12, 384-390.

BARKFR S. B., SUMMERSON W, M., I94I. The colorimetric determination of lactic acid in biological material. J. Biol. Chem, 138, 535.

BARRINGER R., TRENKLE A., BURROUGHS W., I 966 . Effect of protozoa on viable rumen bacteria, digestibility and nitrogen metabolism in sheep. $J$. Anim. Sci., 25, I257 (Abstr.).

BEEDE D. K., FARLIN S. D., 1975. Effect of monensin on in-vitro volatile fatty acid and lactic acid levels. J. Anim. Sci., 41, 390 (Abstr.).

Befson W. M., Perry T. W., Mohler M., Andrews F. N., STOB M., I957. Combinations of an antibiotic and a female hormone for fattening steers. J. Anim. Sci., 16, 845-849.

Brown H., Carroli. I. H., Elitston N. G., Grueter H. P., Mc Askili, J. W., Olson R. D., RATHMACHER R. P., I974. Field evaluation of monensin for improving feed efficiency in feedlot cattle. Pro., western section. American Socicty of Animal Science, 25, 300-302.

Christiansen W. C., Kawashima R., Burroughs W., ig65. Influence of protozoa upon rumen acid production and liveweight gains in lambs. $J$. Anim. Sci., 24, 730-736. 
COLEMAN G. S., I975. The interrelationship between rumen ciliate protozoa and bacteria. p. I49-I64. in Digestion and Metabolism in the Ruminant, Mc DONALD I. W. et WARNER A. C. I. (Ed.). The University of New England publishing unit. Armidale, Australia.

DEMEYER D. I., VAN NEVEI, C. J., I975. Methanogenesis, an integrated part of carbohydrate fermentation, and its control. p. 366-382. In Digestion and Metabolism in the Ruminant. Mc DONALD I. W. et WARNER A.C.I. (ed.). The University of New England publishing unit. Armidale, Australia.

Dixius D. A., Simpsox M. E., 1975. Effects of monensin on digestibility and ruminal microbes. J. Anim. Sci., 41, 398 (Abstr.).

Dinius D. A., Simpsow M. F., Marsh P. B., I976. Fiffect of monensin fed with forage on digestion and the ruminal ecosystem of steers. $J$. Anim. Sci., 42, 229-234.

Dyer I. A., ENSminger M. E., BluE R. L., 1957. Effects of fat, oxytetracycline and stilbestrol on performance and hepatic stores of carotene and vitamin $\mathrm{A}$ in steers. $J$. Anim.Sci., 16, $828-832$.

EADIE J. M., I973. The establishment of rumen ciliate protozoa in sheep fed on whole barley to appetite. Proc. Nutr. Soc., 32, 64 A-65 A.

EADIE J. M., I962. The development of rumen nicrobial populations in lambs and calves under various conditions of management. J. Gen. Microbiol., 29, 563-578.

EADIE J. M., Houson P. N., I962. Effect of the presence or absence of rumen ciliate protozoa on the total rumen bacterial count in lambs. Nature, 193, 503-505.

EAdIE J. M., GIr. J. C., I97 I. The effect of the absence of rumen ciliate protozoa on growing lambs fed on roughage concentrate diet. $B r . J . N u t r ., 26$, I 55-I67.

Eadie J. M., Hyidgaard-Jensen J., Mann S. O,, Reid R. S., Whitei.ilaw F. G., i969. The effect of restricted intake of a barley diet on rumen fermentation in cattle. Proc. Nutr. Soc., 28, 44 A-45 A.

GEAY Y., Berangrir C., I977. Utilisation d'un anticoccidien (monensin) dans la ration des ruminants. I. Effets sur les performances d'engraissement des bovins. Ann. Zootech., 26, 59-68.

HANFy M. E., HoEnN N. M., I967. Monensin, a new biologically active compound. I. Discovery and isolation. Antimicrobial agents and chemotherapy, 349-352.

Howard B. H., 1959. Metabolism of carbohydrates by rumen bacteria. Proc. Nutr. Soc., 18, IO3-IO8.

Harrisson D. G., BEeVER D. E., Thompson D. J., OsboukN D. F., I975. Manipulation of rumen fermentation in sheep by increasing the rate of flow of water from the rumen. J. Agric. Sci., 85, 93-IOI.

Hungate R. E., Fretcher D. W., Dyer I. A., I955. Effects of chlortetracycline feeding on bovine rumen microorganisms. J. Anim. Sci., 14, 997-1002.

HUNGATE R, E., I966. The rumen and its microbes. Londres

Johns A. T., r95 ra. The mecanism of propionic acid formation by propionibacteria. J. Gen. Microbiol., 5, 337-345.

Johns A. T., 195 $\mathrm{r}$. The mecanism of propionic acid formation by "veillonella gazogenes". J. Microbiol., 5, 326-336.

KLOPFENSTEIN T. J., PURSER D. B., TyzNIK W. J., i964. Influence of aureomycin on rumen metabolistn. J. Anim. Sci., 23, 490-495.

Klopfensteis T. J., Purser D. B., Tyznik W. J., I966. Effect of defaunation on feed digestibility, rumen metabolism and blood metabolites. $J$. Anim. Sci., 25, 766-773.

Kolari O. E., Harvey A. I., Meiske, J. C., Aunan W. J., Hansen L. E., ig6o.Diethylstilbestrol, oxytetracycline, linseed oil meal, soybean oil meal, and levels of corn silage in cattle fattening rations. J. Anim. Sci., 19, 104I-I 048.

Kurihara Y., Eadie J. M., Hobson P. N., Mans S. O., I968. Relationship between bacteria and ciliate protozoa in the sheep rumen. J. Gen. Microbiol., 51, 267-288.

MALES J. R., PURSir D. B., I970. Relationship between rumen ammonia levels and the microbial population and volatil fatty acid proportions in faunated and defaunated sheep. Appl. $\mathrm{Micro-}$ biol., 19, $485-489$.

Neuman A. L., SNapl R. R., Gall L. S., I95I. The long time effect of feeding aureomycine to fattening beef cattle, with bacteriological data. J. Anim. Sci., 10, Io58 (Abstr.).

Potter E. L., Coolfy C. O., Richardson L. F., Rathmacher R. P., Raun A. P., i974a. Effect of monensin upon composition of carcass gain of cattle. J. Anim. Sci., 39, 249 (Abstr.).

Potter E. L., Coolfy C. O., Raun A. P., Richarson L. F., Rathmacher R. P., i974b. Effect of monensin on daily gain of cattle on pasture. Proc, western section. American Society of Animal Science, 25, 343 (Abstr.). 
Purser D. B., KLopenstein T. J., Crine J. H., 1965. Influence of Tylosin and Aureomycin upon rumen metabolism and the microbial population. J. Anim. Sci., 24, ro39-1044.

Raun A. P., Cooley C. O., Ratimacher R. P., Richardson L. F., Potrer E. L., 1974. Effect of different levels of monensin on feed efficiency, ruminal and carcass characteristics of cattle. Proc., Western section American Society of Animal Science, 25, 346-347.

Richardson L. F. A., Raun A. P., Potter E. L., Coolfy C. O., Rathmacher R. P., I974. Effect of monensin on ruminal fermentation in-vitro and $i n$-vivo. J. Anim. Sci., 39, $25^{\circ}$ (Abstr.).

Rigaud J., Journer M., 1970. Méthode de dosage des acides gras volatils dans le liquide du rumen. Ann. Biol. anim. Bioch. Biophys, 10, I5I-I57.

SATTER L. D., ESDALE W. J., r968. In-vitro lactate metabolism by ruminal ingesta. Appl. Microbiol, 16, 680-688.

Sauvant D., Gouer Ph., i97o. Comparaison de deux techniques pour dénombrer les protozoaires du rumen et précision obtenue. Ann. Biol. anim. Bioch. Biopliys., 10, 689-696.

Whitelaw F. G., Eadif J. M., Maxi S. O., Reid R. S., I972. Some effects of rumen ciliate protozoa in cattle given restricted amounts of barley diet. Br. J. Nutr., 27, 425-437. 